\title{
Clinical Cancer Research
}

\section{Fragile Histidine Triad Expression in Oral Squamous Cell Carcinoma and Precursor Lesions}

Omar Kujan, Richard Oliver, Luca Roz, et al.

Clin Cancer Res 2006;12:6723-6729. Published online November 22, 2006.

Updated Version Access the most recent version of this article at: doi:10.1158/1078-0432.CCR-06-1475

Cited Articles This article cites 46 articles, 19 of which you can access for free at: http://clincancerres.aacrjournals.org/content/12/22/6723.full.html\#ref-list-1

Citing Articles This article has been cited by 2 HighWire-hosted articles. Access the articles at: http://clincancerres.aacrjournals.org/content/12/22/6723.full.html\#related-urls

E-mail alerts Sign up to receive free email-alerts related to this article or journal.

Reprints and To order reprints of this article or to subscribe to the journal, contact the AACR Subscriptions Publications Department at pubs@aacr.org.

Permissions To request permission to re-use all or part of this article, contact the AACR Publications Department at permissions@aacr.org. 


\title{
Fragile Histidine Triad Expression in Oral Squamous Cell Carcinoma and Precursor Lesions
}

\author{
Omar Kujan, ${ }^{1}$ Richard Oliver, ${ }^{1}$ Luca Roz, ${ }^{4}$ Gabriella Sozzi, ${ }^{4}$ Noel Ribeiro, ${ }^{2}$ Robert Woodwards, ${ }^{2}$ \\ Nalin Thakker, ${ }^{1,3}$ and Philip Sloan ${ }^{1}$
}

\begin{abstract}
Pupose: Fragile histidine triad (FHIT) expression in precursor oral lesions (POL) and oral squamous cell carcinomas (OSCC) was studied with regard to (a) the frequency of loss of FHIT expression, $(b)$ whether loss of FHIT expression correlates with degree of dysplasia in POLs, $(c)$ whether FHIT loss predicts high-risk POLs that are more likely to transform, and $(d)$ whether FHIT loss in OSCCs correlates with survival.

Experimental Design: Ninety-four POLs and 86 OSCCs were immunostained for FHIT. Survival analysis was done for cases with validated clinical outcomes.

Results: By optimizing the immunostaining protocol, we found that FHIT is expressed in a distinctive strong nuclear and weak cytoplasmic pattern in oral tissues. Loss of FHIT expression was found in 42 of $94(45 \%)$ POLs and in 66 of 86 (77\%) OSCCs. We observed a statistically significant positive correlation between frequency of FHIT loss and increasing grade of dysplasia $\left(x^{2}=13.8\right.$; degrees of freedom $\left.=4 ; P=0.008\right)$. Loss of FHITexpression in POLs that progressed to malignancy was more frequent than in those that did not [17 of $25(68 \%)$ versus 12 of 29 $(41.4 \%)$, respectively]. This difference was statistically significant $\left(x^{2}=3.8\right.$; degrees of freedom $=1 ; P=0.046$ ). In OSCCs, loss of FHIT staining indicated a worse prognosis (survival rate, $36.2 \%$ ) than when positive FHIT staining was observed (survival rate, $50 \%$ ), but the difference was not statistically significant $(P=0.546$, Kaplan-Meier, log-rank).

Conclusions: FHIT seems to localize to both nuclear and cytoplasmic domains. FHIT inactivation occurs early in oral carcinogenesis and may be useful molecular marker for progressive dysplastic oral lesions.
\end{abstract}

Oral squamous cell carcinoma (OSCC) is a major cause of morbidity and mortality globally accounting for $\sim 275,000$ new cases and $>120,000$ deaths per annum (1). Moreover, both incidence and mortality rates are still increasing (2) and the overall 5-year survival rate has remained static at $50 \%$ over the last 30 years (3). OSCCs, like many other cancers, show frequent (45-55\%) somatic hemizygous deletions (detected as loss of heterozygosity) on the short arm of chromosome 3 (4). Deletions are also detected in precursor oral lesions (POL), which sometimes precede OSCCs (5). The target of the deletions in one specific region, chromosome band 3p14.2, seems to be the fragile histidine triad (FHIT) gene $(6,7)$. It is now well established that FHIT is an important tumor suppressor gene that is down-regulated in most human cancers

Authors' Affiliations: ${ }^{1}$ School of Dentistry, The University of Manchester; ${ }^{2}$ North Manchester General Hospital; ${ }^{3}$ Medical Genetics, School of Medicine, The University of Manchester, St. Mary's Hospital, Manchester, United Kingdom and ${ }^{4}$ Department of Experimental Oncology, Istituto NazionaleTumori, Milan, Italy Received 6/19/06; revised 8/21/06; accepted 8/31/06.

The costs of publication of this article were defrayed in part by the payment of page charges. This article must therefore be hereby marked advertisement in accordance with 18 U.S.C. Section 1734 solely to indicate this fact.

Requests for reprints: Philip Sloan, School of Dentistry, The University of Manchester, Higher Cambridge Street, Manchester M15 6FH, United Kingdom. Phone: 44-161-275-6788; Fax: 44-161-275-6797; E-mail: p.sloan@ manchester.ac.uk.

(C) 2006 American Association for Cancer Research

doi:10.1158/1078-0432.CCR-06-1475
$(6,8)$. Intragenic deletions and aberrant transcripts have been reported in head and neck SCC and more specifically in OSCC, although there seems to be a disagreement about the frequency of the latter. FHIT promoter methylation associated with loss of FHIT expression has been reported in $\sim 70 \%$ of head and neck tumors and has been shown to correlate with poor outcome $(9-11)$.

In this study, we sought to extend the previous studies by studying FHIT expression in a large series of POLs and OSCCs. We sought to address the following questions. ( $a$ ) What is the frequency of loss of FHIT expression in these lesions from patients from the United Kingdom? (b) Does the loss of FHIT expression correlate with degree of dysplasia in POLs as assessed by the new WHO classification (11)? (c) Can the loss of FHIT predict high-risk POLs that are more likely to progress to malignancy? (d) Does the loss of FHIT expression in carcinomas correlate with survival?

\section{Materials and Methods}

Patients. We obtained ethics approval for the study from a National Health Service Local Research Ethics Committee (reference no. 02/104). Our total study sample series consisted of 190 formalin-fixed, paraffinembedded oral mucosal biopsies from the period 1993 to 2003, which we retrieved from the archives of the Unit of Oral Pathology, Manchester Dental Hospital (Manchester, United Kingdom; ref. Table 1). Two pathologists reviewed all cases to confirm diagnosis; 
Table 1. Number of oral epithelial samples that were enrolled in the study

\section{Type of investigated tissue} No.

Normal oral mucosa

Mild dysplasia

Moderate dysplasia

Severe dysplasia

CIS

Oral epithelial dysplasia cases that showed malignant transformation

Oral epithelial dysplasia cases that showed no OSCC progression OSCC

we graded the degree of dysplasia in POLs according to the WHO 2005 classification (12) and staged the tumors according to the International Union Against Cancer tumor-node-metastasis classification (13) by consensus reporting between two observers (O.K. and P.S.).

We studied three main groups of samples:

1. Group 1: this consisted of 50 cases with 10 specimens each from 5 different categories: normal oral epithelium, POLs with mild, moderate, and severe dysplasia, and carcinoma in situ (CIS).

2. Group 2: this consisted of 54 unifocal POLs with epithelial dysplasia and known clinical outcomes. Of these, 19 cases were diagnosed with mild dysplasia, 14 with moderate dysplasia, 16 with severe dysplasia, and 5 cases as CIS. This set was divided into two main groups according to the clinical outcomes. Twenty-five unrelated cases, in which the patients subsequently had developed invasive OSCC, and 29 unrelated cases, in which the patients did not develop OSCC during the period 1993 to 2004. Follow-up ranged from 2 to 97 months (mean, 29.9 months; SD, 25.4). Of these, $22(40.7 \%)$ patients were female and $32(59.3 \%)$ were males.

3. Group 3: this consisted of 86 OSSCs. Of these, 61 cases were treated with surgery by the same consultant at North Manchester General Hospital (Manchester, United Kingdom) during the period 1993 to 1998 and followed up for at least 5 years (range, 1-102; mean, $\pm 50.8 ;$ SD, \pm 33.4 months). The clinical details of the samples are shown in Table 2 . The overall 5-year survival rate was $39.3 \%$ (range, $34.2-87.7 \%$; mean, $\pm 57.5 \%$; SD \pm 5.1 ). The remaining 25 cases were included in the observational study of FHIT expression but were excluded from the survival analysis.

FHIT immunohistochemistry. An optimization study of FHIT immunostaining was done on human normal oral mucosa $(n=5)$, submandibular salivary gland $(n=5)$, and OSCCs $(n=10)$ accessioned in the routine service but not included in the subsequent study. We used a primary rabbit polyclonal anti-glutathione $S$-transferase-FHIT antibody (Zymed Laboratories, South San Francisco, CA), together with a secondary mouse biotinylated link and a streptavidin-horseradish peroxidase conjugate (DAKO LSAB kit, DAKO, Glostrup, Denmark) with diaminobenzidine as the chromogen. Under optimal conditions with antigen retrieval by microwaving for 15 minutes in $10 \mathrm{mmol} / \mathrm{L}$ citrate buffer ( $\mathrm{pH} \mathrm{6)}$, FHIT was strongly expressed in the nucleus of normal basal and parabasal oral epithelial cells and in 4 of 10 SCCs, accompanied by cytoplasmic staining of variable intensity. In the carcinomas, all stromal cells expressed strong nuclear and moderate cytoplasmic staining. In contrast to previously published studies that reported exclusively cytoplasmic localization of FHIT, we showed a nuclear and cytoplasmic localization of the FHIT protein by a variety of biochemical and immunohistochemical methods. ${ }^{5}$ The optimized method was used to immunostain all of the included cases. The stromal cells were used as a positive internal control and for negative control we omitted the primary antibody.

We categorized FHIT immunoreactivity as 'negative' or 'low expression' (no staining or immunoreactivity staining present in $<10 \%$ of tumor cells) or 'positive' (immunoreactivity in $\geq 10 \%$ of tumor cells) as described previously (9).

Statistical analysis. Kruskall-Wallis, ANOVA, and $\chi^{2}$ tests were used to test the association of FHIT protein expression with different variables of the analyzed lesions. Survival curves for overall survival time were estimated by the Kaplan-Meier test, and the resulting curves were compared using the log-rank test. Multivariate analysis was done

Table 2. The clinicopathologic characteristics of the OSSC patients with known follow-up data

\begin{tabular}{|c|c|}
\hline Characteristics & No. cases $(\%)$ \\
\hline Total number of valid cases & $61(100)$ \\
\hline \multicolumn{2}{|l|}{ Age $(y)$} \\
\hline$\leq 50$ & $2(3.3)$ \\
\hline$>50$ & $59(96.7)$ \\
\hline \multicolumn{2}{|l|}{ Gender } \\
\hline Male & $42(68.9)$ \\
\hline Female & $19(31.1)$ \\
\hline \multicolumn{2}{|l|}{ Stage } \\
\hline I & $13(21.3)$ \\
\hline II & $10(16.4)$ \\
\hline III & $22(36.1)$ \\
\hline IV & $16(26.2)$ \\
\hline \multicolumn{2}{|l|}{ Histologic grading } \\
\hline Well-differentiated SCC & $25(41)$ \\
\hline Moderately differentiated SCC & $17(27.9)$ \\
\hline Poorly differentiated SCC & $18(29.5)$ \\
\hline Basaloid SCC & $1(1.6)$ \\
\hline \multicolumn{2}{|l|}{ Tumor size } \\
\hline $\mathrm{T}_{1}$ & $14(23)$ \\
\hline $\mathrm{T}_{2}$ & $24(39.3)$ \\
\hline $\mathrm{T}_{3}$ & $9(14.8)$ \\
\hline $\mathrm{T}_{4}$ & $14(23)$ \\
\hline \multicolumn{2}{|l|}{ Nodal status } \\
\hline $\mathrm{N}_{0}$ & $38(62.3)$ \\
\hline$N_{1}$ & $14(23)$ \\
\hline $\mathrm{N}_{2}$ & $9(14)$ \\
\hline \multicolumn{2}{|l|}{ Metastases } \\
\hline$M_{0}$ & $54(88.5)$ \\
\hline$M_{1}$ & $7(11.5)$ \\
\hline Recurrence in neck & $15 / 61(24.6)$ \\
\hline Recurrence in primary site & $11 / 61(18)$ \\
\hline New primary tumor & $12 / 61(19.7)$ \\
\hline Death & $37(60.7)$ \\
\hline Oral cancer-related death & $22 / 37(59.5)$ \\
\hline Death related to other diseases & $15 / 37(40.5)$ \\
\hline \multicolumn{2}{|l|}{ Smoking habits* } \\
\hline Smoker & $34(55.7)$ \\
\hline Nonsmoker & $13(21.3)$ \\
\hline Not recorded & $14(23)$ \\
\hline \multicolumn{2}{|l|}{ Alcohol habits* } \\
\hline Drinker & $36(59)$ \\
\hline Nondrinker & $11(18)$ \\
\hline Not recorded & $14(23)$ \\
\hline \multicolumn{2}{|l|}{ Site } \\
\hline Tongue & $26(42.6)$ \\
\hline Floor of mouth & $16(26.2)$ \\
\hline Maxilla & $3(4.9)$ \\
\hline Buccal mucosa & $4(6.6)$ \\
\hline Retromolar trigone & $7(11.5)$ \\
\hline Gingiva/alveolus & $5(8.2)$ \\
\hline
\end{tabular}

*Data are incomplete for all patients.

\footnotetext{
${ }^{5}$ In preparation
} 

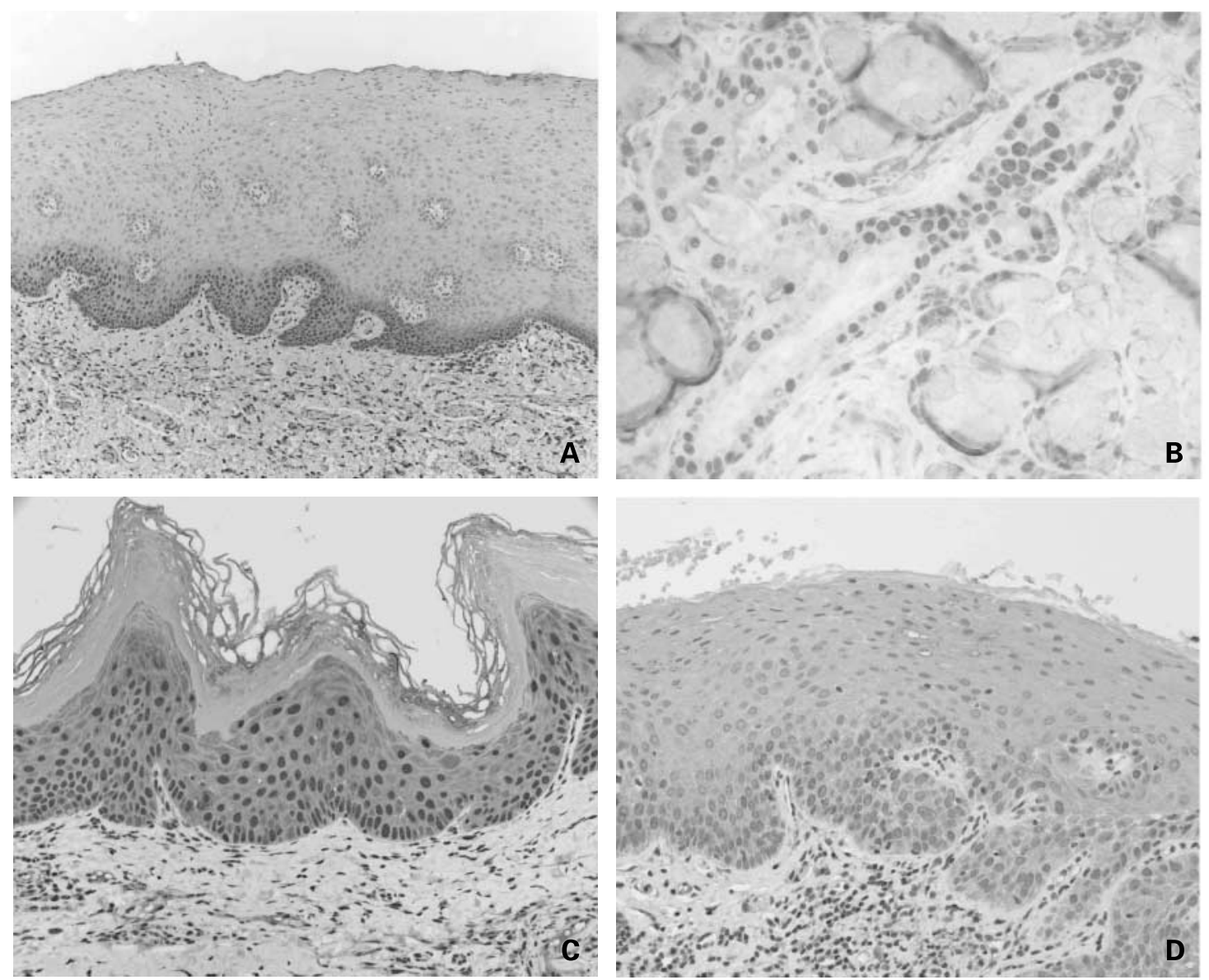

Fig. 1. FHITexpression in oral epithelial tissues. $A$, normal oral squamous epithelium. $B$, ducts of minor salivary gland. $C$, oral epithelial dysplasia $(+)$. $D$, oral epithelial dysplasia (-).

according to Cox proportional hazards model. All tests were two sided. $P<0.05$ was considered to be statistically significant.

\section{Results}

As expected, we observed uniform, strong, positive immunoreactivity for FHIT in normal surface oral epithelium in all cases. This was more prominent in the basal cell layer with the superficial layers showing little or no immunoreactivity. Indeed, a clear demarcation between the stained and non-

Table 3. Frequency of loss of FHIT expression in
normal mucosa, POLs, and OSCCs
\begin{tabular}{lc} 
Lesion & Frequency of loss of \\
& FHIT expression, $\boldsymbol{n} / \boldsymbol{N}(\%)$ \\
\hline Normal oral epithelium & $0 / 10(0)$ \\
POL & $42 / 94(45)$ \\
POL with mild dysplasia & $9 / 29(\sim 31)$ \\
POL with moderate dysplasia & $9 / 24(\sim 38)$ \\
POL with severe dysplasia & $14 / 26(\sim 54)$ \\
POL with CIS & $10 / 15(\sim 67)$ \\
OSCC & $66 / 86(\sim 77)$
\end{tabular}

stained areas was found in all the cases with normal oral squamous epithelium (Fig. 1A). We also observed strong immunoreactivity in excretory ducts of the underlying minor salivary glands (Fig. 1B).

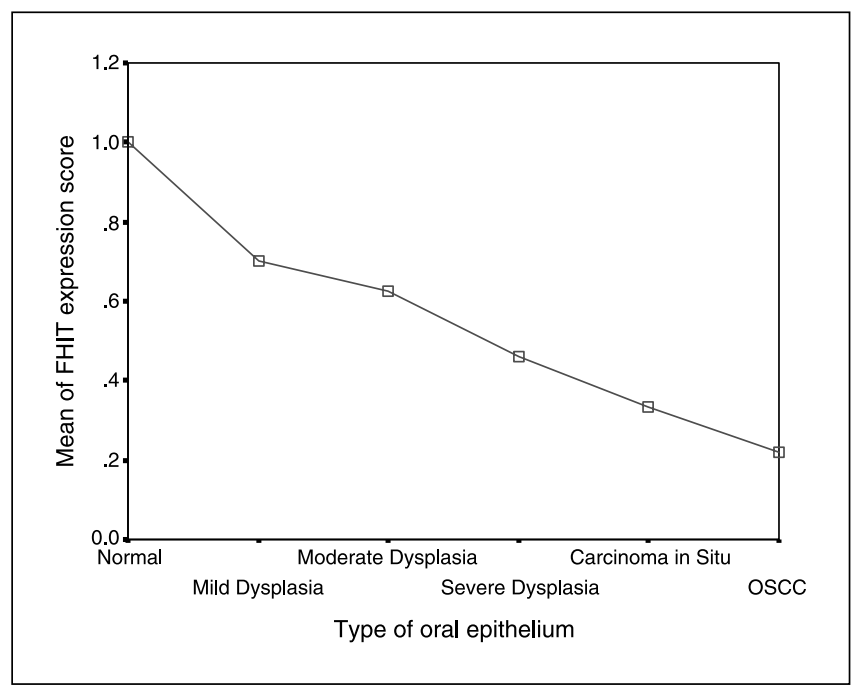

Fig. 2. Analysis of FHIT expression in a model of the natural history of oral carcinogenesis. 


\begin{tabular}{|c|c|c|c|}
\hline & \multicolumn{2}{|c|}{ FHIT expression, $n(\%)$} & \multirow{2}{*}{ Total } \\
\hline & Negative & Positive & \\
\hline Nonprogressive POLs & $12(22.2)$ & $17(31.5)$ & $29(53.7)$ \\
\hline Progressive POLs & $17(31.5)$ & $8(14.8)$ & $25(46.3)$ \\
\hline Total & $29(53.7)$ & $25(46.3)$ & $54(100)$ \\
\hline
\end{tabular}

Altogether, we studied 104 cases (50 from group 1 and 54 from group 2) with variable grades of oral epithelial dysplasia or CIS and normal epithelium. Overall, 42 of 94 (45\%) of POL samples showed reduced or absent FHIT expression (Table 3$)$. We observed a statistically significant $\left(X^{2}=13.8\right.$; degrees of freedom $=4 ; P=0.008$ ) positive correlation between frequency of loss of expression with increasing grade of dysplasia (Fig. 1C and D; Fig. 2; Table 3). Thus, for example, we observed loss of FHIT expression in $\sim 31 \%$ of POLs with mild dysplasia compared with $67 \%$ POLs with CIS. OSCCs showed the highest frequency [66 of $86(\sim 77 \%)]$ of loss of FHIT.

In the progressive and nonprogressive POLs (group 2; Table 4), loss of FHIT expression in POLs that progressed to malignancy was more frequent than in those that did not within the defined period [17 of $25(68 \%)$ versus 12 of 29 (41.4\%), respectively]. This difference was statistically significant $\left(X^{2}=3.8\right.$; degrees of freedom $\left.=1 ; P=0.046\right)$.

In the OSCCs (group 3), we found no association between FHIT expression (Fig. 3A and B) and clinical variables, including age, gender, stage, histologic grading, tumor size, lymph node metastasis, recurrence in neck, recurrence in primary site, and new primary tumor, and the clinical outcomes (Table 5). We were unable to correlate FHIT expression with tobacco or alcohol consumption as complete data were not available.

We also correlated FHIT expression with survival in this group using Kaplan-Meier analysis with log-rank test. The mean follow-up time for the 61 subjects was 50.8 months (range, 1-102 months). At the end of the follow-up period, 37 patients had died.

Twenty-two subjects of 37 had died from oral carcinoma. Moreover, 11 subjects had relapsed by the time of the last follow-up. The survival analysis was done on 61 patients and took into account the following variables: FHIT expression, age, gender, histologic type, tumor size, nodal involvement, metastases, and stage. The overall survival rate was $39.4 \%$. A Kaplan-Meier curve showed that patients with either negative or low expressed FHIT staining had a worse prognosis (survival rate, $36.2 \%$ ) than those of patients with positive FHIT staining (survival rate, 50\%). However, this difference was not statistically significant ( $P=0.546$, log-rank; Fig. 4$)$.

Multivariate analysis was used to assess the independent influence of other prognostic variables on the survival curve. Stage, lymph node metastasis, tumor size, relapse at the primary site, and FHIT expression were included. No statistically significant associations were found between the variables and any significant effect on prognosis, except that relapse at the primary site showed association with poor prognosis (relative risk, $2.561 ; P=0.011$ )

\section{Discussion}

Reduced or absent expression of FHIT has been reported in primary cancers, in cancer cell lines, and in precancerous lesions (reviewed in refs. 6, 8, 14, 15). In addition to hemizygous or homozygous deletions affecting the FHIT gene in cancers, epigenetic changes, such as DNA methylation and histone modification, may lead to decreased expression (reviewed in refs. 16, 17). Immunohistochemistry is a useful method for showing loss of FHIT expression in tumors where FHIT may be inactivated by a variety of molecular mechanisms. Review of published photomicrographs and descriptions of FHIT expression in normal human tissues and cancers shows that exclusively cytoplasmic expression has been regarded as typical $(9,11,18-22)$. Many studies have used various antigen retrieval methods and this has typically produced intense diffuse labeling of the cytoplasm by chromogen $(11,19$, 23-26). Our immunohistochemical optimization study for FHIT staining used antigen retrieval methods, including no pretreatment, proteinase $\mathrm{K}$ digestion, and trypsin digestion. Without pretreatment, only weak nonspecific staining was found. However, proteinase $\mathrm{K}$ or trypsin pretreatment resulted in diffuse cytoplasmic immunoreactivity that was similar to that published in many previous articles $(11,19,23-26)$. In squamous epithelium, all layers of the epithelium were
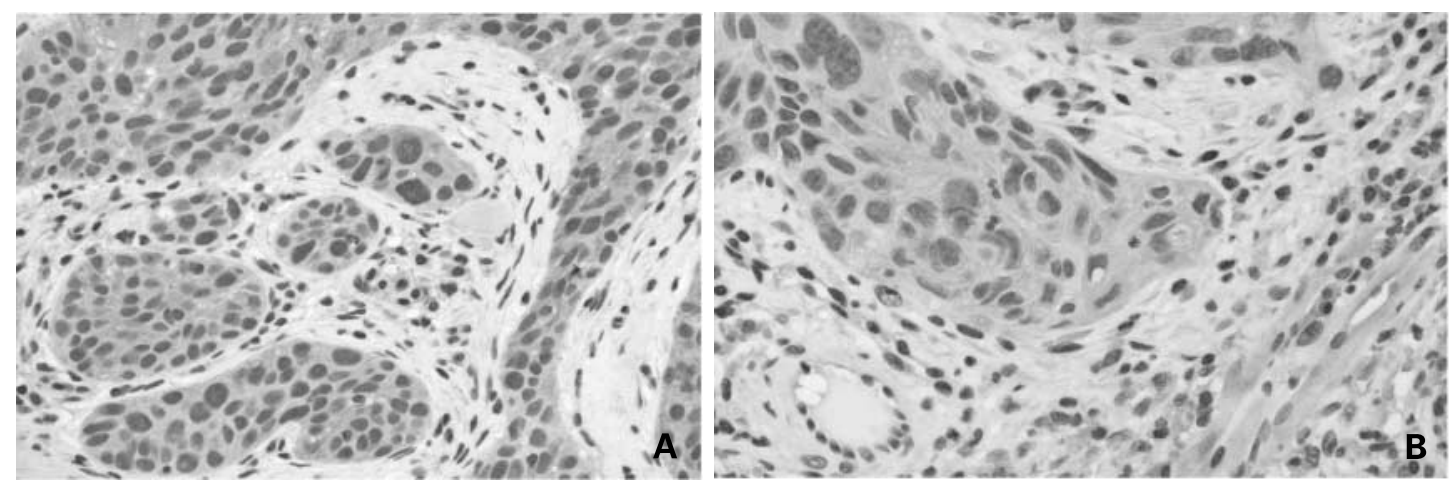

Fig. 3. FHIT expression in OSCC. $A$, OSCC (+). $B$, OSCC (-). 
Table 5. Association between FHIT expression and clinicopathologic variables in OSCC

Factors

Percentage of FHIT expression in tumor cells

\begin{tabular}{ccc}
\hline Negative (<10) & Positive $(\geq 10)$ & $P$ \\
\cline { 3 - 3 }$(\%)$ & $n(\%)$ & \\
\end{tabular}

Age $(y)$

$\leq 50$

$2(3.3)$

Gender

Male

45 (73.7)

$32(52.5)$

Female $15(24.5)$

Stage

I

$12(19.7)$

$9(14.8)$

III

$13(21.3)$

IV

$13(21.3)$

Histologic grading

Well-differentiated SCC

19 (31.1)

$15(24.6)$

$12(19.7)$

Poorly differentiated SCC

1 (1.6)

Basaloid SCC

$13(21.3)$

18 (29.5)

$4(6.5)$

$\mathrm{T}_{2}$

$\mathrm{T}_{3}$

$\mathrm{T}_{4}$

Nodal status

$12(19.7)$

14 (23)

$10(16.4)$

4 (6.6)

1 (1.6)

$1(1.6)$

$9(14.8)$

3 (4.9)

$6(9.9)$

$2(3.2)$

$6(9.9)$

-

$1(1.6)$

$6(9.9)$

$5(8.2)$

$2(3.2)$

$30(49.2)$

$10(16.4)$

8 (13.1)

0.433

$\mathrm{N}_{0}$

$\mathrm{N}_{2}$

7 (11.5)

$4(6.5)$

$2(3.2)$

Metastases

$\begin{array}{lr}M_{0} & 42(68.9) \\ M_{1} & 5(8.2)\end{array}$

$M_{1}$

$10(16.4)$

$10(16.4)$

$9(14.8)$

12 (19.7)

2 (3.2)

$5(8.2)$

$1(1.6)$

3 (4.9)

0.813

Recurrence in primary site

$17(27.9)$

Present status

Alive

$30(49.2)$

$17(27.9)$

Oral cancer-related death

$13(21.3)$

$7(11.5)$

$7(11.5)$

$5(8.2)$

$2(3.2)$

Death related to other diseases

Smoker

Nonsmoker

$9(14.8)$

$9(14.8)$

$4(6.5)$

Alcohol habits*

Drinker

$26(42.6)$

Nondrinker

$8(13.1)$

$10(16.4)$

$3(4.9)$

${ }^{*}$ Data are incomplete for all patients.

intensely labeled, including keratin. However, when we used microwaving in citrate buffer for 15 minutes, we obtained convincing nuclear with weaker cytoplasmic staining and clean negative controls. In addition, a distinctive pattern of labeling that was restricted to the basal and suprabasal cell layers in oral epithelium was seen, consistent with inactivation of FHIT with progressive epithelial maturation. The findings are consistent with reports of FHIT localization in rat tissues where biochemical methodology showed that FHIT was localized to the cytoplasmic membrane and nucleus in normal tissues (27) and in human monocyte cell lineages (28). To our knowledge, our optimization study is the first to show nuclear FHIT immunoreactivity in human epithelial tissues. Further biochemical and immunohistochemical studies in human tissues are in progress to validate this observation.
FHIT and its protein product have been the focus of recent debate with regard to its role in tumorigenesis in bronchial (29), esophageal (30), colorectal (31), breast (32), and cervical (33) cancer. A tumor suppressor role for FHIT was postulated based on the ability of FHIT to eliminate or reduce the tumorigenicity of tumor cells in nude and knockout mice $(34,35)$ and is now widely accepted (6). Several reports showed previously that loss of heterozygosity occurs frequently at the chromosome region containing FHIT $(4,5,36)$ and that the expression of FHIT is frequently altered in head and neck SCC $(11,19,21,37,38)$ and POLs $(6,22,39)$.

In this study, consistent with other studies $(29,39,40)$, we have shown that FHIT is down-regulated early during carcinogenesis and that its down-regulation is associated with increasing dysplasia grade in POLs. In addition, the loss of 


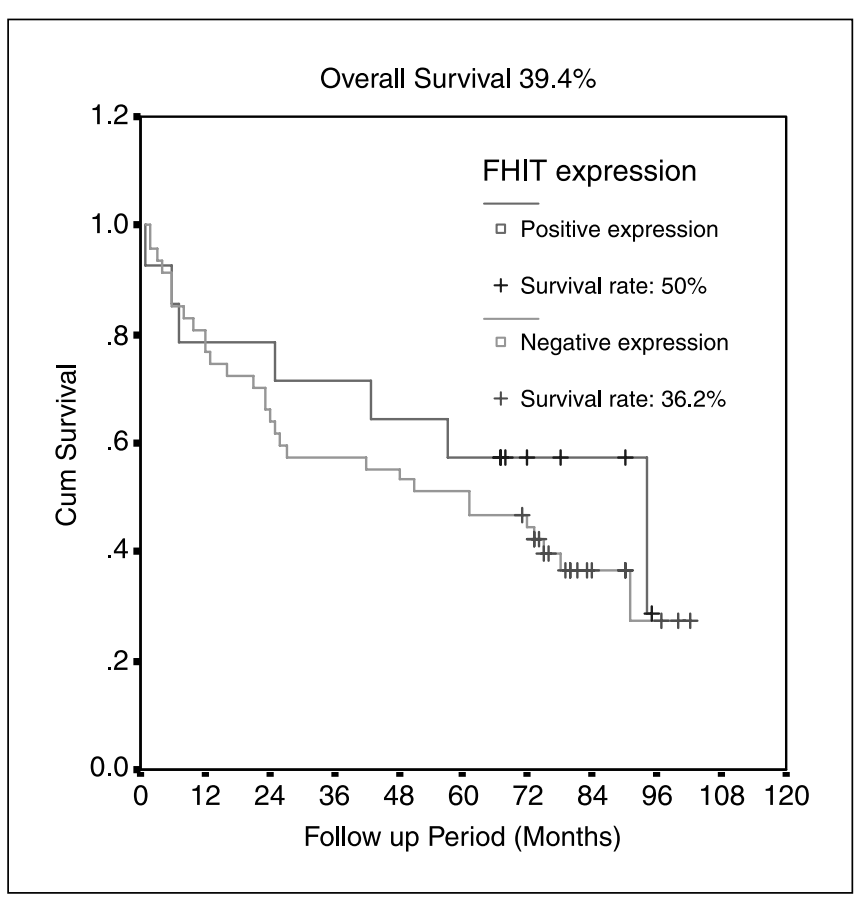

Fig. 4. Kaplan-Meier curves for overall survival stratified according to the level of FHIT expression in patients with OSCC.

FHIT expression was observed more frequently in precursor lesions that developed invasive SCC. More interestingly, a large fraction of SCCs displayed reduced or absent FHIT expression. Studies on cancer evolution showed that genomic alterations that inactivate suppressor genes or activate oncogenes could relieve cells from growth constraints, allowing tumor growth (41). FHIT is a tumor suppressor gene but its pathway is still unclear $(6,15)$. The down-regulation of FHIT may reflect a growth deregulation adding proliferation advantages.

There is a clear need for improved tools to identify, at an early stage, lesions that will develop into OSCC. Study of the natural history of oral cancer and precancer should identify critical stages that can be used to develop preventive strategies or for early detection that may improve the survival rate associated with oral cancer. We attempted to study the role of FHIT in a multistep malignant transformation model of oral carcinogenesis (42). Our results showed that FHIT downregulation is an early frequent event in oral carcinogenesis and may provide a predictor marker for disease progression and outcome alongside other conventional clinicopathologic and molecular markers that are in use. Many molecular markers have been postulated as good predictors $(43,44)$. However, the application of these markers has not yet translated into daily routine service. Current candidate markers lack a clear cutoff point value in determining cases that will transform into malignant disease. We suspect that using a panel of these prognostic markers would help in improving the accuracy of predicting malignant change in POLs.

Our data showed that most invasive OSCCs displayed reduced or absent FHIT expression (76.8\%), which is larger than the findings of other studies. Previous studies showed a range of $4 \%$ to $68 \%$ with absent or markedly reduced FHIT expression in OSSC $(9-11,18,20,21,37)$. This discrepancy may depend on the specificity and sensitivity related to different FHIT antibodies, staining protocol, scoring system, and tissue collection.

A few studies have shown that absent or low levels of FHIT protein are associated with poor prognosis (reduced overall survival time) or with advanced stage in OSSC (9-11). We noted that FHIT inactivation was associated with worse prognosis in OSSC but the effect was not statistically significant. In addition, we did not find any correlation between FHIT expression levels and clinicopathologic variables, such as degree of differentiation, lymph node status, and stage. Similar findings were presented by Mineta et al. (20) and Paradiso et al. (21). In addition, the multivariate Cox regression model failed to find any significant correlation between FHIT expression and any prognostic variables, except that the relapse at the primary site showed association with poor prognosis (relative risk, 2.561; $P=0.011$ ), which is a common finding in OSCC.

In the literature, there is controversy about the prognostic significance of FHIT in primary OSCC. This dispute originates from studies using either immunohistochemistry or molecular techniques that are mostly based on heterogeneous samples of OSCCs $(10,20,21)$. It can be argued that the best method to evaluate FHIT expression in a primary tumor is immunohistochemistry because the proportion of cells exhibiting the protein, as well as their level of expression, can be determined and the method can be easily used in practice $(10,18,20)$.

\section{Conclusions}

Our observations of the loss of FHIT protein expression in a substantial percentage of oral precursor and cancerous lesions suggest that FHIT alterations play an important role in the development of oral cancer. FHIT inactivation may be useful for predicting malignant changes in biopsies of oral precursor lesions. Cells collected from brushing the oral mucosa could also be a valuable tool for the surveillance of oral dysplasia lesions (45). Moreover, due to the high frequency of FHIT inactivation in oral carcinogenesis and the successful restoration of FHIT in cell lines and mice $(35,46-48)$, the FHIT gene could present an eligible target for gene therapy for POLs and for prevention of OSCC.

\section{Acknowledgments}

We thank Susan Donnelly for her help with the laboratory work.

\section{References}

1. Parkin DM, Bray F, Ferlay J, Pisani P. Global cancer statistics, 2002. CA Cancer J Clin 2005;55:74-108.

2. La Vecchia $C$, Lucchini F, Negri $E$, Levi F. Trends in oral cancer mortality in Europe. Oral Oncol 2004; 40:433-9.
3. Brunin F, Mosseri V, Jaulerry C, Point D, Cosset JM, Rodriguez J. Cancer of the base of the tongue: past and future. Head Neck 1999;21: $751-9$.

4. Wu CL, Sloan P, Read AP, Harris R, Thakker N. De- letion mapping on the short arm of chromosome 3 in squamous cell carcinoma of the oral cavity. Cancer Res 1994;54:6484-8.

5. Roz L, Wu CL, Porter S, et al. Allelic imbalance on chromosome $3 p$ in oral dysplastic lesions: an early 
event in oral carcinogenesis. Cancer Res 1996;56: 1228-31.

6. Campiglio M, Bianchi F, Andriani F, et al. Diadenosines as FHIT-ness instructors. J Cell Physiol 2006; 208:274-81.

7. Rosin MP, LamWL, Poh C, et al. 3p14 and 9p21 loss is a simple tool for predicting second oral malignancy at previously treated oral cancer sites. Cancer Res 2002;62:6447-50

8. lliopoulos D, Guler G, Han SY, et al. Roles of FHIT and WWOX fragile genes in cancer. Cancer Lett 2006; 232:27-36.

9. Lee Jl, Soria JC, Hassan K, et al. Loss of Fhit expression is a predictor of poor outcome in tongue cancer. Cancer Res 2001;61:837-41.

10. Guerin LA, Hoffman HT, Zimmerman MB, Robinson RA. Decreased fragile histidine triad gene protein expression is associated with worse prognosis in oral squamous carcinoma. Arch Pathol Lab Med 2006; 130:158-64.

11. Tai SK, Lee JI, Ang KK, et al. Loss of Fhit expression in head and neck squamous cell carcinoma and its potential clinical implication. Clin Cancer Res 2004; 10:5554-7.

12. Barnes L, Eveson J, Reichart P, Sidransky D. World Health Organization classification of tumors. Pathology and genetics of tumors of the head and neck. Lyon: IARC Press; 2005. p. 177-80.

13. Sobin LH, Wittekind C, International Union Against Cancer. TNM classification of malignant tumors. 6th ed. New York: Wiley-Liss; 2002. p. 22-6.

14. Ishii $H$, Ozawa K, Furukawa Y. Alteration of the fragile histidine triad gene early in carcinogenesis: an update. J ExpTher Oncol 2003;3:291 -6.

15. Pekarsky Y, Zanesi N, Palamarchuk A, Huebner K, Croce CM. FHIT: from gene discovery to cancer treatment and prevention. Lancet Oncol 2002;3: $748-54$.

16. Hsieh $\mathrm{CL}$, Jones PA. Meddling with methylation. Nat Cell Biol 2003;5:502-4.

17. Lee EJ, Lee BB, Kim JW, et al. Aberrant methylation of fragile histidine triad gene is associated with poor prognosis in early stage esophageal squamous cell carcinoma. Eur J Cancer 2006;42: $972-80$.

18. van Heerden WF, Swart TJ, Robson B, et al. FHIT RNA and protein expression in oral squamous cell carcinomas. Anticancer Res 2001;21:2425-8.

19. van Heerden WF, Swart TJ, van Heerden MB, Pekarsky Y, Sutherland R, Huebner K. Fhit protein expression in oral epithelium: immunohistochemical evaluation of three antisera. Anticancer Res 2001;21: 2419-23.

20. Mineta H, Miura K, Takebayashi S, et al. Low expression of fragile histidine triad gene correlates with high proliferation in head and neck squamous cell carcinoma. Oral Oncol 2003;39:56-63.

21. Paradiso A, Ranieri G, Stea B, et al. Altered p16INK4a and Fhit expression in carcinogenesis and progression of human oral cancer. Int J Oncol 2004; 24:249-55

22. Chang KW, Kao SY,Tzeng RJ, et al. Multiple molecular alterations of FHIT in betel-associated oral carcinoma. J Pathol 2002;196:300-6.

23. Wali A, Srinivasan R, Shabnam MS, Majumdar $S$, Joshi K, Behera D. Loss of fragile histidine triad gene expression in advanced lung cancer is consequent to allelic loss at 3p14 locus and promoter methylation. Mol Cancer Res 2006;4:93-9.

24. Holschneider $\mathrm{CH}$, Baldwin RL, Tumber K, Aoyama C, Karlan BY. The fragile histidine triad gene: a molecular link between cigarette smoking and cervical cancer. Clin Cancer Res 2005;11:5756-63.

25. Huang LW, Chao SL, Chen TJ. Reduced Fhit expression in cervical carcinoma: correlation with tumor progression and poor prognosis. Gynecol Oncol 2003,90:331 - 7

26. Yang $Q$, Yoshimura G, Suzuma T, et al. Clinicopathological significance of fragile histidine triad transcription protein expression in breast carcinoma. Clin Cancer Res 2001;7:3869-73.

27. Golebiowski F, Kowara R, Pawelczyk T. Distribution of Fhit protein in rat tissues and its intracellular localization. Mol Cell Biochem 2001;226: 49-55.

28. Zhao P, Hou N, Lu Y. Fhit protein is preferentially expressed in the nucleus of monocyte-derived cells and its possible biological significance. Histol Histopathol 2006;21:915-23.

29. Sozzi G, Pastorino U, Moiraghi L, et al. Loss of FHIT function in lung cancer and preinvasive bronchial lesions. Cancer Res 1998;58:5032-7.

30. ShimadaY, Sato F, Watanabe G, et al. Loss of fragile histidine triad gene expression is associated with progression of esophageal squamous cell carcinoma, but not with the patient's prognosis and smoking history. Cancer 2000;89:5-11.

31. Hao XP, Willis JE, Pretlow TG, et al. Loss of fragile histidine triad expression in colorectal carcinomas and premalignant lesions. Cancer Res 2000;60: $18-21$.

32. Gatalica Z, Lele SM, Rampy BA, Norris BA. The expression of Fhit protein is related inversely to disease progression in patients with breast carcinoma. Cancer 2000;88:1378-83

33. Birrer MJ, Hendricks D, FarleyJ, et al. Abnormal Fhit expression in malignant and premalignant lesions of the cervix. Cancer Res 1999;59:5270-4.

34. Huebner K, DruckT, Siprashvili Z, Croce CM, Kovatich A, McCue PA. The role of deletions at the FRA3B/
FHIT locus in carcinogenesis. Recent Results Cancer Res 1998;154:200-15.

35. Dumon KR, Ishii H, Fong LY, et al. FHITgene therapy prevents tumor development in Fhit-deficient mice. Proc Natl Acad Sci U S A 2001;98:3346-51.

36. el-Naggar AK, Hurr K, Batsakis JG, Luna MA, Goepfert H, Huff V. Sequential loss of heterozygosity at microsatellite motifs in preinvasive and invasive head and neck squamous carcinoma. Cancer Res 1995;55:2656-9.

37. Virgilio L, Shuster M, Gollin SM, et al. FHIT gene alterations in head and neck squamous cell carcinomas. Proc Natl Acad Sci U S A 1996;93: $9770-5$.

38. Kannan K, Munirajan AK, Bhuvarahamurthy V, et al. FHIT gene mutations and single nucleotide polymorphism in Indian oral and cervical squamous cell carcinomas. Oral Oncol 2000;36:189-93.

39. Tanimoto $\mathrm{K}$, Hayashi $\mathrm{S}$, Tsuchiya $\mathrm{E}$, et al. Abnormalities of the FHIT gene in human oral carcinogenesis. $\mathrm{Br}$ J Cancer 2000;82:838-43.

40. Kitamura A, Yashima K, Okamoto $E$, et al. Reduced Fhit expression occurs in the early stage of esophageal tumorigenesis: no correlation with p53 expression and apoptosis. Oncology 2001;61:205-11.

41. Hanahan D, Weinberg RA. The hallmarks of cancer. Cell 2000;100:57-70.

42. Perez-Ordonez B, Beauchemin M, Jordan RC. Molecular biology of squamous cell carcinoma of the head and neck. J Clin Pathol 2006:59:445-53.

43. Grandis JR, Tweardy DJ. Elevated levels of transforming growth factor $\alpha$ and epidermal growth factor receptor messenger RNA are early markers of carcinogenesis in head and neck cancer. Cancer Res 1993:53 3579-84.

44. Lo Muzio L, Pannone G, Leonardi R, et al. Survivin a potential early predictor of tumor progression in the oral mucosa. J Dent Res 2003;82:923-8.

45. Kujan O, Desai M, Sargent A, Bailey A, Turner A, Sloan P. Potential applications of oral brush cytology with liquid-based technology: results from a cohort of normal oral mucosa. Oral Oncol 2006 42:810-8.

46. Roz L, Gramegna M, Ishii H, Croce CM, Sozzi G Restoration of fragile histidine triad (FHIT) expression induces apoptosis and suppresses tumorigenicity in lung and cervical cancer cell lines. Proc Natl Acad Sci U S A 2002;99:3615-20.

47. Ishii $H$, Vecchione $A$, Fong $L Y$, et al. Cancer prevention and therapy in a preclinical mouse model: impact of FHIT viruses. Curr GeneTher 2004;4:53-63.

48. Pichiorri F, Trapasso F, PalumboT, et al. Preclinical assessment of FHIT gene replacement therapy in human leukemia using a chimeric adenovirus, Ad5/F35 Clin Cancer Res 2006;12:3494-501. 